\title{
Mortality from lung cancer among Sardinian patients with silicosis
}

\author{
Plinio Carta, Pier Luigi Cocco, Duilio Casula
}

\begin{abstract}
The mortality of 724 subjects with silicosis, first diagnosed in 1964-70 in the Sardinia region of Italy, was followed up through to 31 December 1987. Smoking, occupational history, chest $x$ ray films, and data on lung function were available from clinical records for each member of the cohort. The overall cohort accounted for 10956.5 person-years. The standardised mortality ratios (SMRs) for selected causes of death (International Classification of Diseases (ICD) eighth revision) were based on the age specific regional death rates for each calendar year. An excess of deaths for all causes (SMR = 1.40) was found, mainly due to chronic obstructive lung disease, silicosis, and tuberculosis with an upward trend of the SMR with increasing severity of the International Labour Office (ILO) radiological categories. Twenty two subjects died from lung cancer $(S M R=1 \cdot 29,95 \%$ confidence interval $(95 \% \mathrm{CI})=0 \cdot 8-2 \cdot 0)$. The risk increased after $a 10$ and 15 year latency but the SMR never reached statistical significance. No correlation was found between lung cancer and severity of the radiological category, the type of silica (coal or metalliferous mines, quarries etc), or the degree of exposure to silica dust. A significant excess of deaths from lung cancer was found among heavy smokers $(S M R=4 \cdot 11)$ and subjects with airflow obstruction (SMR $=\mathbf{2 \cdot 8 3}$ ). A nested case-control study was planned to investigate whether the association between lung cancer and airway obstruction was due to confounding by smoking. No association was found with the ILO categories of silicosis or the estimated cumulative exposure to silica. The risk estimate for lung cancer by airflow obstruction after adjusting by cigarette consumption was $\mathbf{2 . 8 6}$ for a mild impairment and $\mathbf{7 \cdot 2 3}$ for a
\end{abstract}

Institute of Occupational Medicine, University of Cagliari, Sardinia, Italy

P Carta, P L Cocco, D Casula severe obstruction. The results do not show any clear association between exposure to silica, severity of silicosis, and mortality from lung cancer. Other environmental or individual factors may act as confounders in the association between silicosis and lung cancer. Among them, attention should be given to chronic airways obstruction as an independent risk factor for lung cancer in patients with silicosis.

Contradictory published results prevent conclusive statements about the possible association between exposure to silica dust, silicosis, and lung cancer.

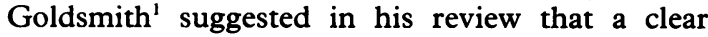
relation existed between exposure to free crystalline silica, or silicosis, and lung cancer. Maillard ${ }^{2}$ and Heppleston ${ }^{3}$ in reviews of studies in animals and man came to the opposite conclusion and considered that the reported association between silicosis and lung cancer might be attributed to confounders such as cigarette smoking or concomitant occupational exposures other than silica. A recent International Agency for Research on Cancer monograph ${ }^{4}$ stated that the evidence of carcinogenicity for crystalline silica was "sufficient" in experimental studies, but "limited" in man. ${ }^{4}$ Some experimental studies in rats found an association between the fibrogenic reaction to exposure to silica and lung cancer. ${ }^{56}$ Other studies in mice, however, did not show any differences between treatment and control. ${ }^{7}$ Furthermore, Stenback et $\mathrm{al}^{8}$ showed a significant increase in incidence of lung cancer in Syrian hamsters only after combining silica with benzo(a)pyrene. Saffiotti suggested that perhaps cell mediators released during the fibrogenic process might cause the hyperplasia of epithelial cells and tumours seen in the lungs of rats treated with silica. ${ }^{9}$

Much of the epidemiological evidence of an association between exposure to respirable silica and lung cancer is derived from cohort or case control studies on patients with silicosis ${ }^{10-17}$ and a possible link between silicosis, but not exposure to silica by itself, and lung cancer has frequently been reported. Previous epidemiological data ${ }^{18-20}$ showed that, even after adjusting for smoking, lung cancer occurred 
more frequently in patients affected by chronic obstructive pulmonary disease with hypersecretion of mucus and airflow limitation. Choen et $a^{2122}$ and Beaty et al ${ }^{23}$ suggested that airway obstruction was an independent risk factor for bronchogenic carcinoma in the general population.

Carta $e$ e al $^{24}$ found a non-significant $10 \%$ excess of lung cancer mortality in a 14 year follow up of a cohort of Sardinian subjects with silicosis; a significant excess of deaths from lung cancer was found, independently of the severity of silicosis, only among smokers and patients with airways obstruction.

The present paper extends this follow up study to 31 December 1987 and investigates the association between lung cancer and several risk factors using a nested case-control approach.

\section{Subjects and methods}

All cases of silicosis who were less than 75 years old (724) among about 6000 male workers admitted to the Institute of Occupational Medicine of the University of Cagliari (Sardinia, Italy) during the period January 1964-December 1970 entered the cohort. This institute was the only referral centre for all claims for silicosis submitted to the three Sardinian provincial offices of the National Institute for Insurance of Occupational Diseases. Thus the cohort includes all incident cases of silicosis in Sardinia during 1964-70.

The same medical staff assessed the diagnosis with a standard procedure. The standard chest $x$ ray films of all members of the cohort were blindly reviewed according to the International Labour Office criteria (ILO 1980) for the 12 point classification scale of pneumoconiosis. ${ }^{25}$ Of all films, $154(21.3 \%)$ were classified between categories $1 / 0$ and $1 / 2,205$ $(28.3 \%)$ between $2 / 1$ and $2 / 3,289(39.9 \%)$ between $3 / 2$ and $3 / 3$, and $76(10.5 \%)$ between $A$ and $B$ categories.

Detailed occupational histories, routinely collected at the time of admission, were available from the clinical records. All subjects had worked in dusty industries in Sardinia, mainly lead and zinc metalliferous mines, coal mines, and granite quarries. Three hundred and thirty one men $(45.7 \%)$ had only worked in Sardinian metalliferous mines in which the silica content in respirable dust was higher than $7 \%$. One hundred subjects $(13.8 \%)$ were miners from Sardinian lead and zinc mines with lower silica content (less than $2 \%$ ). One hundred and nineteen subjects $(16.5 \%)$ had only worked in Sardinian coal mines with a mean silica content in respirable dust of around $3 \%$. Fifty three patients $(7 \cdot 3 \%)$ were granite quarrymen and 121 subjects $(16 \cdot 7 \%)$ had a mixed occupational history in these working areas.

Previous studies on the work environment and health conditions in Sardinian mines and other dusty industries provided data on exposure to respirable dust and silica content. ${ }^{26-28}$ These data and the duration of employment in each workplace allowed a categorical estimate of cumulative silica exposure for each subject. A worklife cumulative index of exposure to silica was calculated for each subject by multiplying the mean respirable silica in each workplace (respirable dust $\left(\mathrm{mg} / \mathrm{m}^{3}\right) \times \%$ of silica) by the estimated hours of exposure per year of work. The cumulative exposure to silica was reported as $\mathrm{gh} / \mathrm{m}^{3}{ }^{28}{ }^{29}$ The distribution of subjects within classes of cumulative silica exposure was: low $(n=216$; $29.8 \%)$; intermediate $(\mathrm{n}=301 ; 41.6 \%)$; high $(\mathrm{n}=207 ; 28.6 \%)$.

Only 23 subjects were still at work when silicosis was diagnosed, suggesting a minimal underestimation of the worklife cumulative exposure to silica.

Direct interviews at the time of admission also provided information on smoking habits and mean number of cigarettes smoked per day. Two hundred and fifty four men $(35.1 \%)$ were classified as never smokers, 109 subjects (15.0\%) stopped smoking before entering the study and 361 patients $(49.9 \%)$ were still current smokers at the start of follow up; no changes in smoking habits were recorded during follow up.

Spirometric data at the first diagnosis of silicosis were available in 690 patients $(95.3 \%)$. The slow vital capacity (VC) and the ratio between forced expiratory volume in one second $\left(\mathrm{FEV}_{1}\right)$ and VC $\left(\mathrm{FEV}_{1} / \mathrm{VC}\right)$ were compared with the mean predicted values from the reference summary equations of the European Coal and Steel Community. ${ }^{30} \mathrm{~A}$ restrictive or obstructive impairment was defined by comparing the observed data with normal limits (1.64 standard deviations from reference regressions). A reduced VC was seen in 302 patients $(43.8 \%)$, and an abnormal $\mathrm{FEV}_{1} / \mathrm{VC}$ ratio was found in $318(46 \cdot 1 \%)$.

\section{COHORT STUDY}

Vital state on 31 December 1987 was ascertained through the Insurance Institute and the residence town registers. Ten subjects $(1 \cdot 38 \%)$ not traced after 31 December 1977 because of emigration were considered alive and contributed to person-years at risk up to this date. A copy of the death certificate was available for all deceased subjects. The underlying cause of death was coded according to the eighth revision of the International Classification of Diseases. ${ }^{31}$ The reliability of death certificates was checked, for each case, by the analysis of the Insurance Institute periodical medical controls. The sex, five year age class, and calendar year specific regional death rates were available for the period 1969-83. Expected deaths for 1964-9 and 1983-7 were computed applying the regional death rates found in 1969 and 1983 to the total person-years at risk during these two periods. Person-years since 
first admission, expected deaths from specific cause and standardised mortality ratios (SMRs) were calculated using a modified computing program for MS-DOS personal computer. ${ }^{32}$ The approximate $95 \%$ confidence intervals (95\% CIs) for the SMRs were calculated according to Rothman. ${ }^{33}$

\section{NESTED CASE CONTROL STUDY FOR LUNG CANCER}

Possible risk factors for lung cancer were also studied with a nested case control study. Cases were subjects who had died from lung cancer. Four controls were randomly selected for each case from the set of persons at risk at the time of death of each case, ${ }^{34}$ matching for year of birth ( \pm two years), year of entry ( \pm two years) and smoking habit (never smokers, former smokers, and current smokers). Controls who died of chronic respiratory diseases not concerned with tumours were excluded and replaced with other subjects chosen at random in the same subset.

The Mantel-Haenszel stratified analysis ${ }^{35}$ was used to estimate the association between category of pneumoconiosis, exposure to silica, and impairment of lung function as independent risk factors, and lung cancer. The $95 \%$ CIs were calculated according to Miettinen. ${ }^{36}$

\section{Results}

COHORT STUDY

Table 1 shows the main characteristics of the overall cohort by ILO categories of silicosis. The 724 patients with silicosis followed up since their hospital admission accounted for 10956.5 person years. The highest ILO category had the highest age at entry, the lowest mean survival time, and the highest death rate.

The mean duration of employment in dusty jobs was shortest among patients with confluent silicosis.
The estimated index of cumulative exposure to silica dust, on the contrary, showed an upward trend with severity of silicosis and it was highest in those patients in the ILO category A-B.

The frequency of airflow obstruction $\left(\mathrm{FEV}_{1} / \mathrm{VC}\right.$ ratio below $90 \%$ of the predicted value) increased significantly with the severity of silicosis and was highest among patients with confluent silicosis, despite the proportion of smokers being lowest in this group.

Table 2 shows the results of the cause specific mortality analysis. Total deaths $(n=438 ; 60.3 \%)$ were significantly more than expected, mainly due to excess deaths for silicotuberculosis and non-neoplastic chronic respiratory diseases (chronic bronchitis, emphysema, complicated silicosis). Among the other non-malignant causes of death, a significant twofold risk was found for chronic diseases of the urinary system.

The mortality from all cancers was slightly lower than expected. Only cancers of the lips and pharynx had a significantly higher than expected SMR (four observed deaths $v$ one expected death; 95\% $\mathrm{CI}=1 \cdot 6-9.9)$. All four patients were smokers. A non-significant $29 \%$ increase of the SMR for lung cancer was found among the entire cohort (22 observed $v 17$ expected deaths). Table 3 reports the SMRs for some selected causes of death according to latency periods since the diagnosis of silicosis. No trend was found for all deaths. The mortality for all cancers increased with the latency, but the SMR was always below one. The excess of lung cancer deaths increased up to $53 \%$ with a 15 year latency assumption but this was not statistically significant.

Table 4 shows the mortality for lung cancer by ILO categories and daily smoking of cigarettes. The SMR did not show a trend by severity of silicotic nodule profusion; the most severe category had the

Table 1 Characteristics of the cohort by ILO categories (follow up 1964-87)

\begin{tabular}{|c|c|c|c|c|c|}
\hline & \multicolumn{4}{|c|}{ ILO categories } & \multirow[b]{2}{*}{ Total } \\
\hline & $1 / 0-1 / 2$ & $2 / 1-2 / 3$ & $3 / 2-3 / 3$ & $A-B$ & \\
\hline $\begin{array}{l}\text { No of subjects } \\
\text { Person-years }\end{array}$ & $\begin{array}{c}154 \\
2492 \cdot 5\end{array}$ & $\begin{array}{l}205 \\
3131 \cdot 5\end{array}$ & $\begin{array}{c}289 \\
4231 \cdot 5\end{array}$ & $\begin{array}{c}76 \\
1001 \cdot 1\end{array}$ & $\begin{array}{c}724 \\
10956 \cdot 5\end{array}$ \\
\hline $\begin{array}{l}\text { Mean age at admission }(y) \\
\text { Mean year of entry }(y) \\
\text { Mean year at first exposure }(y) \\
\text { Mean years of silica exposure }(y) \\
\text { Mean cumulative exposure }{ }^{\star}\end{array}$ & $\begin{array}{r}54 \cdot 5 \\
1966 \cdot 3 \\
1931 \cdot 1 \\
24 \cdot 3 \\
7 \cdot 7\end{array}$ & $\begin{array}{r}55 \cdot 1 \\
1966 \cdot 2 \\
1932 \cdot 1 \\
24 \cdot 4 \\
8 \cdot 1\end{array}$ & $\begin{array}{r}56 \cdot 8 \\
1966 \cdot 3 \\
1931 \cdot 6 \\
24 \cdot 0 \\
8 \cdot 3\end{array}$ & $\begin{array}{r}59 \cdot 6 \\
1966 \cdot 1 \\
1929 \cdot 1 \\
22 \cdot 5 \\
8 \cdot 7\end{array}$ & $\begin{array}{r}56 \cdot 1 \\
1966 \cdot 3 \\
1931 \cdot 4 \\
24 \cdot 0 \\
8 \cdot 3\end{array}$ \\
\hline $\begin{array}{l}\text { No of smokers }(\%) \dagger \\
1-14 \mathrm{cig} / \text { day }(\%) \dagger \\
\geqslant 15 \mathrm{cig} / \text { day }(\%) \dagger \\
\text { Airflow obstruction }(\%)\end{array}$ & $\begin{array}{l}68 \cdot 2 \\
54 \cdot 6 \\
13 \cdot 6 \\
39 \cdot 9\end{array}$ & $\begin{array}{l}69 \cdot 8 \\
58 \cdot 0 \\
11 \cdot 8 \\
45 \cdot 5\end{array}$ & $\begin{array}{r}62 \cdot 6 \\
53 \cdot 6 \\
9 \cdot 0 \\
47 \cdot 5\end{array}$ & $\begin{array}{r}54 \cdot 0 \\
44 \cdot 7 \\
9 \cdot 3 \\
56 \cdot 5\end{array}$ & $\begin{array}{l}64 \cdot 9 \\
54 \cdot 1 \\
10 \cdot 8 \\
46 \cdot 2\end{array}$ \\
\hline $\begin{array}{l}\text { No of deaths } \\
\text { Death rate }(\%) \\
\text { Mean age at death }(y) \\
\text { Mean survival time }(y)\end{array}$ & $\begin{array}{l}83 \\
53 \cdot 9 \\
70 \cdot 9 \\
17 \cdot 6\end{array}$ & $\begin{array}{r}117 \\
57 \cdot 1 \\
68 \cdot 9 \\
16 \cdot 5\end{array}$ & $\begin{array}{r}184 \\
63 \cdot 7 \\
70 \cdot 5 \\
15 \cdot 8\end{array}$ & $\begin{array}{l}54 \\
71 \cdot 0 \\
71 \cdot 2 \\
14 \cdot 1\end{array}$ & $\begin{array}{r}438 \\
60 \cdot 5 \\
70 \cdot 2 \\
16 \cdot 2\end{array}$ \\
\hline
\end{tabular}

ॠEstimated cumulative exposure to respirable silica $\left(\mathrm{gh} / \mathrm{m}^{3}\right)$.

†Former and current smokers. 
Table 2 Cause specific SMRs for 724 Sardinian patients with silicosis (follow up 1964-87)

\begin{tabular}{|c|c|c|c|c|}
\hline Cause of death & Obs $†$ & $\operatorname{Exp} \dagger$ & $S M R$ & $95 \% C I$ \\
\hline All causes & 438 & $312 \cdot 7$ & $1 \cdot 40^{\star}$ & $1 \cdot 28-1 \cdot 54$ \\
\hline All cancers & 63 & $68 \cdot 7$ & 0.92 & $0 \cdot 72-1 \cdot 17$ \\
\hline $\begin{array}{l}\text { Specific cancers: } \\
\text { Buccal, pharynx } \\
\text { Stomach } \\
\text { Lung } \\
\text { Urinary tract } \\
\text { Leukaemia, lymphomas } \\
\text { All others }\end{array}$ & $\begin{array}{r}4 \\
8 \\
22 \\
11 \\
3 \\
15\end{array}$ & $\begin{array}{r}1 \cdot 0 \\
8 \cdot 3 \\
17 \cdot 0 \\
10 \cdot 1 \\
2 \cdot 4 \\
12 \cdot 7\end{array}$ & $\begin{array}{l}4 \cdot 00^{\star} \\
0 \cdot 97 \\
1 \cdot 29 \\
1 \cdot 09 \\
1 \cdot 25 \\
1 \cdot 18\end{array}$ & $\begin{array}{l}1.61-9.89 \\
0.48-1.93 \\
0.85-1.96 \\
0.60-1.97 \\
0.40-3.88 \\
0.71-1.96\end{array}$ \\
\hline $\begin{array}{l}\text { Tuberculosis } \\
\text { Diseases of circulatory system } \\
\text { Diseases of respiratory system } \\
\text { Diseases of digestive system } \\
\text { Diseases of urinary system }\end{array}$ & $\begin{array}{r}31 \\
76 \\
222 \\
26 \\
9\end{array}$ & $\begin{array}{r}2 \cdot 6 \\
88 \cdot 9 \\
32 \cdot 2 \\
24 \cdot 0 \\
4 \cdot 4\end{array}$ & $\begin{array}{l}11 \cdot 92^{\star} \\
0 \cdot 87 \\
6 \cdot 90^{\star} \\
1 \cdot 07 \\
2 \cdot 04^{\star}\end{array}$ & $\begin{array}{l}9 \cdot 05-15 \cdot 71 \\
0 \cdot 64-1 \cdot 17 \\
6 \cdot 16-7 \cdot 72 \\
0 \cdot 73-1 \cdot 58 \\
1 \cdot 07-3 \cdot 87\end{array}$ \\
\hline All accidents & 11 & $13 \cdot 7$ & $0 \cdot 80$ & $0 \cdot 44-1 \cdot 47$ \\
\hline
\end{tabular}

${ }^{\star} \mathrm{p}<0.05$

†Obs = observed deaths; $\operatorname{Exp}=$ expected deaths.

lowest value. On the contrary an upward trend from lung cancer mortality was found with increasing cigarette smoking within each radiological category. A fourfold excess of deaths from lung cancer was found in heavy smokers (more than 14 cigarettes per day). No synergic effect on SMR, however, between smoking and the severity of nodule profusion in the

Table 3 Selected SMRs by latency

\begin{tabular}{|c|c|c|c|}
\hline & \multicolumn{3}{|l|}{ Latency $(y)$} \\
\hline & 5 & 10 & 15 \\
\hline Person-years & $9319 \cdot 0$ & $4929 \cdot 0$ & 2391.5 \\
\hline $\begin{array}{l}\text { All causes: } \\
\text { Obs/Exp deaths } \\
\text { SMR }(95 \% \mathrm{CI})\end{array}$ & $\begin{array}{l}348 / 254 \cdot 2 \\
1 \cdot 37(1 \cdot 2-1 \cdot 5)\end{array}$ & $\begin{array}{l}237 / 165 \cdot 4 \\
1 \cdot 43(1 \cdot 3-1 \cdot 6)\end{array}$ & $\begin{array}{l}105 / 80 \cdot 8 \\
1 \cdot 30(1 \cdot 1-1 \cdot 6)\end{array}$ \\
\hline $\begin{array}{l}\text { All cancers: } \\
\text { Obs/Exp deaths } \\
\text { SMR }(95 \% \mathrm{CI})\end{array}$ & $\begin{array}{l}47 / 57 \cdot 5 \\
0.82(0 \cdot 6-1 \cdot 1)\end{array}$ & $\begin{array}{l}39 / 39 \cdot 7 \\
0.98(0.7-1 \cdot 3)\end{array}$ & $\begin{array}{l}21 / 21.2 \\
0.99(0.6-1.5)\end{array}$ \\
\hline $\begin{array}{l}\text { Lung cancer: } \\
\text { Obs/Exp deaths } \\
\text { SMR }(95 \% \mathrm{CI})\end{array}$ & $\begin{array}{l}19 / 14 \cdot 8 \\
1 \cdot 29(0 \cdot 8-2 \cdot 0)\end{array}$ & $\begin{array}{l}16 / 10 \cdot 7 \\
1 \cdot 49(0 \cdot 9-2 \cdot 4)\end{array}$ & $\begin{array}{l}9 / 6.0 \\
1.53(0.8-2.9)\end{array}$ \\
\hline
\end{tabular}

chest $x$ ray films was found. No association was seen between risk of lung cancer and duration of exposure. Although the lowest SMR for lung cancer was in the low category of cumulative exposure to silica with the shortest latency, the risk for lung cancer did not seem to be associated with increasing exposure to silica, and the SMRs were not significantly different, even allowing for a 15 years latency (table 5 ).

Table 6 reports the main characteristics of 690 patients with available spirometry. Subjects with $\mathrm{FEV}_{1} / \mathrm{VC}$ ratios equal to or higher than $90 \%$ of the predicted value were considered free from airflow obstruction. Subjects with $\mathrm{FEV}_{1} / \mathrm{VC}$ ratios below this cut off point were considered as having an airways obstruction, moderate if the values ranged from $80-89 \%$ of the predicted value and a severe impairment if below $80 \%$. About half the patients with available spirometry had an airflow obstruction, moderate for $22.5 \%$ and severe for $23.6 \%$ of them. Subjects with impaired $\mathrm{FEV}_{1} / \mathrm{VC}$ ratios were older at entry and included a higher proportion of smokers than those free from airflow obstruction. Also, the proportion of the most severe radiological forms

Table 4 Lung cancer mortality by cigarette smoking and ILO categories

\begin{tabular}{|c|c|c|c|c|c|c|c|c|c|c|c|c|}
\hline \multirow[b]{3}{*}{ Smoking category } & \multicolumn{9}{|c|}{ ILO categories } & \multirow{2}{*}{\multicolumn{3}{|c|}{ Total }} \\
\hline & \multicolumn{3}{|c|}{$1 / 0-1 / 2$} & \multicolumn{3}{|c|}{$2 / 1-2 / 3$} & \multicolumn{3}{|c|}{$>3 / 2$} & & & \\
\hline & Obs & $\operatorname{Exp}$ & $S M R$ & Obs & $\operatorname{Exp}$ & $S M R$ & Obs & $\operatorname{Exp}$ & $S M R$ & Obs & $\operatorname{Exp}$ & $S M R$ \\
\hline Never smokers & \multirow{4}{*}{\multicolumn{2}{|c|}{$\begin{array}{l}1 \\
(0 \cdot 1-5 \cdot 1) \ddagger \\
3 \quad 2 \cdot 2 \\
(0 \cdot 4-4 \cdot 2) \\
2 \quad 0 \cdot 4 \\
(1 \cdot 3-17 \cdot 7) \\
6 \quad 4 \cdot 0 \\
(0 \cdot 7-3 \cdot 3)\end{array}$}} & 0.73 & $\underline{0}$ & 1.5 & - & \multirow{4}{*}{\multicolumn{2}{|c|}{$\begin{array}{l}3 \quad 3 \cdot 0 \\
(0 \cdot 3-3 \cdot 1) \\
2 \quad 4 \cdot 4 \\
(0 \cdot 1-1 \cdot 8) \\
3 \quad 0 \cdot 9 \\
(1 \cdot 2-9 \cdot 8) \\
8 \quad 8 \cdot 2 \\
(0 \cdot 5-1 \cdot 9)\end{array}$}} & 1.00 & \multirow{4}{*}{\multicolumn{2}{|c|}{$\begin{array}{l}{ }^{4} \quad 5 \cdot 9 \\
(0 \cdot 3-1 \cdot 8) \\
11 \quad 9 \cdot 4 \\
(0 \cdot 6-2 \cdot 1) \\
7 \quad 1 \cdot 7 \\
(2 \cdot 1-8 \cdot 1) \\
22 \quad 17 \cdot 0 \\
(0 \cdot 8-2 \cdot 0)\end{array}$}} & 0.69 \\
\hline $1-14 \mathrm{cig} /$ day $\dagger$ & & & 1.37 & $\begin{array}{l}6 \\
(1 \cdot 0\end{array}$ & 6) & $2 \cdot 10$ & & & 0.46 & & & $1 \cdot 16$ \\
\hline$\geqslant 15 \mathrm{cig} /$ day $\dagger$ & & & $4 \cdot 46^{\star}$ & 2 & $\begin{array}{l}0.4 \\
7.4)\end{array}$ & $4 \cdot 99 \star$ & & & $3 \cdot 41^{\star}$ & & & $4 \cdot 11^{\star}$ \\
\hline Total & & & 1.51 & $\begin{array}{l}8 \\
(0.8-\end{array}$ & $\begin{array}{l}4 \cdot 8 \\
3)\end{array}$ & 1.67 & & & 0.97 & & & $1 \cdot 29$ \\
\hline
\end{tabular}

${ }^{\star} \mathrm{p}<0.05$.

†Includes former and current smokers.

$+95 \%$ CI. 
Table 5 Lung cancer mortality by cumulative silica exposure

\begin{tabular}{|c|c|c|c|c|c|c|c|c|c|}
\hline & \multicolumn{9}{|c|}{ Cumulative exposure to silica dust } \\
\hline & \multicolumn{3}{|l|}{ Low } & \multicolumn{3}{|c|}{ Intermediate } & \multicolumn{3}{|l|}{ High } \\
\hline & Obs & $\operatorname{Exp}$ & $S M R$ & Obs & $\operatorname{Exp}$ & $S M R$ & Obs & $\operatorname{Exp}$ & $S M R$ \\
\hline \\
\hline 0 & \multirow{3}{*}{$\begin{array}{l}4 \\
(0 \cdot 3-2 \cdot 3)^{\star} \\
3 \\
(0 \cdot 2-2 \cdot 3) \\
2 \\
(0 \cdot 3-5 \cdot 3)\end{array}$} & $4 \cdot 7$ & 0.85 & \multirow{3}{*}{$\begin{array}{l}10 \\
(0 \cdot 8-2 \cdot 7) \\
9 \\
(0 \cdot 8-2 \cdot 9) \\
3 \\
(0 \cdot 4-6 \cdot 0)\end{array}$} & $6 \cdot 9$ & $1 \cdot 45$ & \multirow{3}{*}{$\begin{array}{l}8 \\
(0 \cdot 7-2 \cdot 9) \\
7 \\
(0 \cdot 7-3 \cdot 0) \\
4 \\
(0 \cdot 6-4 \cdot 1)\end{array}$} & 5.5 & 1.46 \\
\hline 5 & & $4 \cdot 0$ & 0.75 & & $6 \cdot 0$ & 1.51 & & $4 \cdot 8$ & $1 \cdot 44$ \\
\hline 15 & & 1.5 & 1.32 & & $2 \cdot 3$ & 1.29 & & 2.6 & 1.54 \\
\hline
\end{tabular}

$\star 95 \% \mathrm{CI}$.

(classified as $3 / 2$ or more) increased with the increasing category of airflow obstruction.

Table 7 reports the SMRs for selected causes of death by category of obstructive airflow impairment. Whereas silicotic patients with a normal $\mathrm{FEV}_{1} / \mathrm{VC}$ ratio showed low risk for lung cancer $(S M R=0.54$; 95\% CI $0 \cdot 22-1 \cdot 28$ ), the observed deaths for lung neoplasms were significantly more than expected among the patients with airflow obstruction. In fact,

Table 6 Characteristics of cohort by lung function ${ }^{\star}$

\begin{tabular}{lccc}
\hline & \multicolumn{3}{c}{$F E V_{t} / V C(\%$ predicted $)$} \\
\cline { 2 - 4 } & $\geqslant 90$ & $89-80$ & $<80$ \\
\hline Number of subjects & 372 & 155 & 163 \\
Person-years & 5852.5 & 2319.0 & 2069.2 \\
Mean age at entry (y) & 54.8 & 56.7 & 57.9 \\
Mean years of exposure (y) & 24.3 & 23.8 & 23.9 \\
No of smokers (\%) $\dagger$ & 63.4 & 71.3 & 71.4 \\
ILO Category 3/2 or more (\%) & 47.2 & 50.0 & 57.3 \\
\hline
\end{tabular}

*Only patients with available spirometry.

tIncludes former and current smokers. the SMR for lung cancer increased with the severity of the airways obstruction, from a twofold excess in moderate obstruction, to about a threefold excess in subjects with severe reduction of the $\mathrm{FEV}_{1} / \mathrm{VC}$ ratio. An upward trend of mortality with degree of airflow obstruction was also found for other causes of death.

NESTED CASE-CONTROL STUDY FOR LUNG CANCER

Table 8 shows the characteristics of the 22 cases of lung cancer and 88 randomly selected matched controls.

Cases and controls differed only in the $\mathrm{FEV}_{1} / \mathrm{VC}$ ratio, which was significantly lower among cases. Seventeen cases $(77 \cdot 3 \%)$ and 38 controls $(43 \cdot 1 \%)$ had an obstructive impairment at admission. The latency between the entry to study and death from lung cancer among cases was 12.5 years (SD 6.1) (range 2-22).

Table 9 shows the odds ratios (ORs) for ILO radiological categories of silicosis and cumulative exposure to silica. This confirmed that lung cancer was not significantly related either to the severity of profusion of radiological opacities or the category of

Table 7 Selected SMRs by airflow obstruction ( $A O$ )

\begin{tabular}{|c|c|c|c|c|c|c|c|c|c|}
\hline \multirow[b]{3}{*}{ Cause of death } & \multicolumn{9}{|l|}{$F E V_{1} / V C$} \\
\hline & \multicolumn{3}{|c|}{$\begin{array}{l}\geqslant 90 \% \text { predicted } \\
\text { (no } A O)\end{array}$} & \multicolumn{3}{|c|}{$\begin{array}{l}89-80 \% \text { predicted } \\
\text { (moderate } A O)\end{array}$} & \multicolumn{3}{|c|}{$\begin{array}{l}<80 \% \text { predicted } \\
(\text { severe } A O)\end{array}$} \\
\hline & Obs & $\operatorname{Exp}$ & $S M R$ & Obs & $\operatorname{Exp}$ & $S M R$ & Obs & $\operatorname{Exp}$ & $S M R$ \\
\hline All causes & $\begin{array}{l}184 \\
(1 \cdot 0-1 \cdot 3) \dagger\end{array}$ & $162 \cdot 9$ & $1 \cdot 13$ & $\begin{array}{l}90 \\
(1 \cdot 0-1 \cdot 5)\end{array}$ & $74 \cdot 3$ & $1 \cdot 21$ & $\begin{array}{l}120 \\
(1 \cdot 7-2 \cdot 4)\end{array}$ & $59 \cdot 3$ & $2 \cdot 02^{\star}$ \\
\hline All cancers & $\begin{array}{l}26 \\
(0.5-1 \cdot 1)\end{array}$ & $36 \cdot 4$ & 0.74 & $\begin{array}{l}15 \\
(0 \cdot 6-1 \cdot 6)\end{array}$ & $15 \cdot 6$ & 0.96 & $\begin{array}{l}15 \\
(0.7-1.9)\end{array}$ & $13 \cdot 0$ & $1 \cdot 15$ \\
\hline Lung cancer & $\begin{array}{c}5 \\
(0 \cdot 2-1 \cdot 3)\end{array}$ & $9 \cdot 2$ & 0.54 & $\begin{array}{l}8 \\
(1 \cdot 1-4 \cdot 2)\end{array}$ & $3 \cdot 8$ & $2 \cdot 12^{\star}$ & $\stackrel{9}{(1 \cdot 5-5 \cdot 3)}$ & $3 \cdot 2$ & $2 \cdot 83^{\star}$ \\
\hline $\begin{array}{l}\text { Diseases of } \\
\text { respiratory } \\
\text { system }\end{array}$ & $\begin{array}{l}93 \\
(4 \cdot 6-6 \cdot 6)\end{array}$ & $16 \cdot 8$ & $5 \cdot 50^{\star}$ & $\begin{array}{l}46 \\
(4 \cdot 7-7 \cdot 8)\end{array}$ & $7 \cdot 6$ & $6.02^{\star}$ & $\begin{array}{l}64 \\
(8 \cdot 6-12 \cdot 8)\end{array}$ & $6 \cdot 1$ & $10 \cdot 52^{\star}$ \\
\hline $\begin{array}{l}\text { Diseases of } \\
\text { circulatory } \\
\text { system }\end{array}$ & $\begin{array}{l}33 \\
(0 \cdot 5-1 \cdot 1)\end{array}$ & $43 \cdot 3$ & $0 \cdot 76$ & $\begin{array}{l}14 \\
(0 \cdot 5-1 \cdot 3)\end{array}$ & $18 \cdot 1$ & 0.77 & $\begin{array}{l}19 \\
(0 \cdot 8-2 \cdot 0)\end{array}$ & $14 \cdot 5$ & 1.31 \\
\hline
\end{tabular}

$\star \mathrm{p}<0.05$

$+95 \% \mathrm{CI}$. 
cumulative exposure to silica dust. On the contrary, a significant increase of the lung cancer risk was associated with the detection of airflow obstruction (table 10).

Table 8 Nested case-control study for lung cancer: characteristics of cases and controls

\begin{tabular}{|c|c|c|c|}
\hline & Cases & Controls & p Values \\
\hline $\begin{array}{l}\text { No } \\
\text { Age at entry }(\mathrm{y}(\mathrm{SD})) \\
\text { Years of exposure }(\mathrm{y}(\mathrm{SD})) \\
\text { Cumulative exposure }\left(\mathrm{gh} / \mathrm{m}^{3}\right) \\
\text { ILO Category } 3 / 2 \text { or more }(\%) \\
\text { Cigarettes/day } \\
\text { FEV } / \mathrm{VC}(\% \text { predicted) } \\
\text { Abnormality in } \mathrm{FEV} / \mathrm{VC}(\%)\end{array}$ & $\begin{array}{l}22 \\
49 \cdot 6(12 \cdot 7) \\
25 \cdot 1(10 \cdot 4) \\
8 \cdot 4(4 \cdot 1) \\
36 \cdot 4 \\
12 \cdot 2(4 \cdot 6) \\
79 \cdot 8(16 \cdot 8) \\
77 \cdot 3\end{array}$ & $\begin{array}{l}88 \\
51 \cdot 2(8 \cdot 5) \\
23 \cdot 6(9 \cdot 4) \\
7 \cdot 9(4 \cdot 6) \\
50 \cdot 0 \\
10 \cdot 4(5 \cdot 2) \\
89 \cdot 9(11 \cdot 3) \\
43 \cdot 1\end{array}$ & $\begin{array}{l}N^{*} S^{\star} \\
N^{\star} \\
N^{\star} \\
N^{\star} \\
N^{\star} \\
0 \cdot 01^{\star} \\
0.01 \dagger\end{array}$ \\
\hline
\end{tabular}

«Unpaired $t$ test.

†Yates' $\chi^{2}$ test.

Table 9 Distribution of cases and controls and risk estimates for lung cancer by ILO categories and cumulative exposure to silica dust

\begin{tabular}{|c|c|c|c|c|}
\hline & Cases & Controls & Crude OR & Adjusted $O R^{\star}$ \\
\hline $\begin{array}{l}\text { ILO categories: } \\
1 / 0-1 / 2 \\
2 / 1-2 / 3 \\
3 / 2 \text { or more }\end{array}$ & $\begin{array}{l}6 \\
8 \\
8\end{array}$ & $\begin{array}{l}19 \\
25 \\
44\end{array}$ & $\begin{array}{l}1.00 \\
1.01(0.9-1 \cdot 1) \dagger \\
0.57(0 \cdot 2-1 \cdot 7)\end{array}$ & $\begin{array}{l}1.00 \\
0.94(0.8-1 \cdot 1) \\
0.65(0.3-1.4)\end{array}$ \\
\hline $\begin{array}{l}\text { Estimated } \\
\text { cumulative } \\
\text { silica exposure: } \\
\text { Low } \\
\text { Intermediate } \\
\text { High }\end{array}$ & $\begin{array}{r}5 \\
10 \\
7\end{array}$ & $\begin{array}{l}31 \\
33 \\
24\end{array}$ & $\begin{array}{l}1.00 \\
1.87(0.5-7.3) \\
1.81(0.5-6.4)\end{array}$ & $\begin{array}{l}1.00 \\
1.95(0.4-10.1) \\
1.86(0.4-8.6)\end{array}$ \\
\hline
\end{tabular}

* Mantel-Haenszel stratified analysis by cigarette consumption. $+95 \% \mathrm{CI}$.

Table 10 Distribution of cases and controls and risk estimates for lung cancer by airflow obstruction and cigarette consumption

\begin{tabular}{|c|c|c|c|c|}
\hline & \multicolumn{3}{|c|}{$F E V, / V C$ (\% predicted) } & \multirow[b]{2}{*}{ Total } \\
\hline & $\geqslant 90$ & $89-80$ & $<80$ & \\
\hline $\begin{array}{l}\text { Never smokers } \\
\text { Cases } \\
\text { Controls }\end{array}$ & $\begin{array}{l}2 \\
9\end{array}$ & $\begin{array}{l}0 \\
5\end{array}$ & $\begin{array}{l}2 \\
2\end{array}$ & $\begin{array}{r}4 \\
16\end{array}$ \\
\hline $\begin{array}{l}\text { 1-14 cig/day: } \\
\text { Cases } \\
\text { Controls }\end{array}$ & $\begin{array}{r}2 \\
33\end{array}$ & $\begin{array}{r}5 \\
16\end{array}$ & $\begin{array}{l}4 \\
8\end{array}$ & $\begin{array}{l}11 \\
57\end{array}$ \\
\hline $\begin{array}{l}\geqslant 15 \mathrm{cig} / \text { day: }{ }^{\star} \\
\text { Cases } \\
\text { Controls }\end{array}$ & $\begin{array}{l}1 \\
8\end{array}$ & $\begin{array}{l}2 \\
4\end{array}$ & $\begin{array}{l}4 \\
3\end{array}$ & $\begin{array}{r}7 \\
15\end{array}$ \\
\hline $\begin{array}{l}\text { Total: } \\
\text { Cases } \\
\text { Controls }\end{array}$ & $\begin{array}{r}5 \\
50\end{array}$ & $\begin{array}{r}7 \\
25\end{array}$ & $\begin{array}{l}10 \\
13\end{array}$ & $\begin{array}{l}22 \\
88\end{array}$ \\
\hline Crude OR & 1.00 & $\begin{array}{l}2 \cdot 80 \\
(1 \cdot 6-5 \cdot 0) \dagger\end{array}$ & $\begin{array}{l}7 \cdot 69 \\
(2 \cdot 4-24 \cdot 7)\end{array}$ & \\
\hline Adjusted OR + & 1.00 & $\begin{array}{l}2 \cdot 86 \\
(1 \cdot 5-5 \cdot 4)\end{array}$ & $\begin{array}{l}7 \cdot 23 \\
(2 \cdot 2-24 \cdot 1)\end{array}$ & \\
\hline
\end{tabular}

*Includes former and current smokers.

$+95 \%$ CI.

$\ddagger$ Mantel-Haenszel stratified analysis by cigarette smoking.
The smoking adjusted risk estimate was 2.86 for moderate airflow obstruction and 7.23 for severe airflow obstruction. The detection of a restrictive impairment, expressed by a reduced VC, was only weakly associated with lung cancer. The risk estimate was 1.23 (CI 0.9-1.9) for VC between 83 and $70 \%$ of the predicted value (moderate restriction) and 2.45 (CI $0 \cdot 6-9 \cdot 2$ ) for more severe restriction.

\section{Discussion}

The data showed no association between exposure to silica dust or severity of radiological silicosis and lung cancer.

The cohort, which accounted for all cases of silicosis during 1964-70, provided a good description of Sardinian workers heavily exposed to silica dust in Sardinian mines and quarries.

No official data on exposure to radon were available. Sporadic recent measurements in some mines suggest a low level of exposure on average, but inconsistencies in the measurements prevent reliable estimates. Diesel engines were introduced in our mines only after 1970, so the risk factor of exposure to diesel fuel cannot be considered for the cohort.

The radiological diagnosis of silicosis was made blindly by persons unaware of the occupational history and degree of compensation of subjects. Only subjects whose chest films were classified $1 / 0$ or more were included in the study. No further information on changes in radiological state, smoking habits, and lung function during the follow up were recorded.

The results confirm our previous study ${ }^{24}$ and agree with the conclusions of a recent necropsy casecontrol study among South African white gold miners, ${ }^{37}$ whose lung cancer risk was not associated with severity of silicosis or cumulative exposure to silica.

Several studies of men compensated for silicosis reported lung cancer risks to be significantly raised by some two to fourfold. ${ }^{10-17}$ Most of these studies, however, included workers from various mining industries and foundries with a number of potential occupational confounders, such as radon daughters, diesel emissions, and polycyclic aromatic hydrocarbons; these occupational exposures were seldom considered. Moreover, because of poor environmental data, the estimation of exposure to silica dust was generally reported as years of employment in different working areas. Also, none of the cited papers reported data about the severity of the radiological types of pneumoconiosis. Studies based on compensation lists might include not only patients with silicosis, diagnosed by chest $x$ ray film, but also subjects without evidence of nodular pneumoconiosis who were affected by other respiratory diseases related either to occupational dust exposure or cigarette smoking, such as chronic obstructive 
pulmonary diseases. Also, most of the cited studies were not able to control for confounding by smoking. When data on cigarette smoking were available, the results indicated an increased risk of lung cancer only in patients with silicosis who smoked. ${ }^{12} 1417$

On the other hand, some authors did not find any increase in risk of lung cancer either among large cohorts of British coalminers or in patients with pneumoconiosis. ${ }^{38-40}$

Although respirable dust in British coalmines contained on average $4.5 \%$ of free crystalline silica, ${ }^{41}$ environmental exposure to radon daughters was estimated as low..$^{42}$ The case-control study of Ames also failed to find any association between coal workers' pneumoconiosis and lung cancer risk among American coalminers. ${ }^{43}$

The hypothesis of a relation between exposure to respirable silica and lung cancer was investigated among granite cutters, a working population with relatively pure exposure to silica. The results were contradictory. Negative evidence was provided by Davis et $\mathrm{al}^{44}$ and by the proportional mortality study of Steenland and Beaumont. ${ }^{45}$ Other studies ${ }^{4647}$ on the contrary, found increased mortality from lung cancer in granite workers and suggested exposure to silica as an aetiological factor in the initiation or promotion of malignant neoplasms. Data on smoking habits were available for only one cohort. ${ }^{47}$

The possible association between airflow obstruction and incidence of lung cancer was first pointed out by Choen and coworkers. ${ }^{21-23}$ More recently, this hypothesis has been supported by the results of a nested case-control study among workers heavily exposed to asbestos. ${ }^{48}$ The study of Skillrud et al ${ }^{49}$ and the results of the Johns Hopkins Lung Project ${ }^{50}$ concluded that airways obstruction substantially increased the risk of lung cancer after controlling for age and smoking. Thus airways obstruction appears to be an independent risk factor for lung cancer that is more important than age and smoking. ${ }^{51}$

Possible mechanisms by which chronic airway obstruction might amplify the individual lung cancer risk include a decreased clearance of inhaled carcinogens with enhancement of their potential effectiveness on lung tissue..$^{22} 48$

The significant increase in the SMR for lung cancer found in our study among subjects with airflow obstruction suggested the opportunity of controlling the possible confounding by smoking. A nested case-control study was conducted, matching lung cancer cases to controls by year of birth, year of entry into the cohort, and smoking habit, and further adjusting the ORs by number of cigarettes smoked per day. We found a strong association between lung cancer and the severity of airways obstruction, independent of smoking and severity of radiological silicosis. Exposure to dust is known to be a common risk factor for pneumoconiosis and chronic obstruc- tive lung disease. The high prevalence of severe obstructive impairments, commonly seen among patients with silicosis or in dust exposed workers, mostly among smokers, may be an often unmeasured but important confounding factor in the relation between silica, silicosis, and lung cancer.

$\mathrm{McDonald}^{52}$ suggested that the evidence of carcinogenicity of crystalline silica in man is not proved and that many confounders should be taken into account in the supposed relation between lung cancer and silicosis. As well as smoking and radon exposure, attention must be given to the role of chronic airflow obstruction as an independent factor enhancing the risk for lung cancer in workers exposed to silica dust and other well known carcinogens.

1 Goldsmith DF, Guidotti TL, Johnston DR. Does occupational exposure to silica cause lung cancer? Am J Ind Med 1982; 3:423-40.

2 Maillard JM. Silicose et cancer bronchique. Poumon-Coeur 1980;36:41-8.

3 Heppleston AG. Silica, pneumoconiosis and carcinoma of the lung. Am J Ind Med 1985;7:285-94.

4 International Agency for Research on Cancer. Monographs on the evaluation of the carcinogenic risk of chemicals to humans. Vol 42. Silica and some silicates. Lyon: IARC, 1987.

5 Dagle GE, Wehner AP, Clark ML, Buschbom RL. Chronic inhalation exposure of rats to quartz. In: Goldsmith DF, Winn DM, Shy CM, eds. Silica, silicosis and cancer: controversy in occupational medicine. New York: Praeger, 1986:255-66. (Cancer research monographs, Vol 2.)

6 Groth DH, Stettler LE, Platek SF, Lal JB, Burg JR. Lung tumors in rats treated with quartz by intratracheal instillation. In: Goldsmith DF, Winn DM, Shy CM, eds. Silica, silicosis and cancer: controversy in occupational medicine. New York: Praeger, 1986:243-54. (Cancer research monographs, Vol 2.)

7 Wilson T, Scheuchenzuber WJ, Eskew ML, Zankower A. Comparative pathological aspects of chronic olivine and silica inhalation in mice. Environ Res 1986;39:331-44.

8 Stenback F, Wasenius VM, Rowland J. Alveolar and interstitial changes in silica-associated lung tumors in Syrian hamsters. In: Goldsmith DF, Winn DM, Shy CM, eds. Silica, silicosis and cancer: controversy in occupational medicine. New York: Praeger, 1986:199-214. (Cancer research monograph, Vol 2.)

9 Saffiotti U. Attivita' cancerogena della silice e possibile ruolo patogenetico della reazione cellulare fibrogena. Med Lav 1985;76:351-7.

10 Westerholm P. Silicosis-observation on a case register. Scand J Work Environ Health 1980;6:1-86.

11 Finkelstein M, Kusiak R, Suranyi G. Mortality among miners receiving workmens' compensation for silicosis in Ontario: 1940-75. J Occup Med 1982;24:663-7.

12 Zambon P, Mastrangelo G, Saia B, Braga M, Crepet $M$. Exposure to silica and lung cancer, a case control study. Scand $J$ Work Environ Health 1983;9:70-1.

13 Gudbergsson H, Kurppa K, Koskinen H, Vasama M. An association between silicosis and lung cancer. A register approach. In: Proceedings of the VIth international conference on pneumoconiosis, Bochum. Geneva: International Labour Organisation, 1983:212-6.

14 Forastiere F, Lagorio S, Michelozzi P, et al. Silica, silicosis and lung cancer among ceramic workers: a case-referent study. $\mathrm{Am}$ $J$ Ind Med 1986;10:363-70.

15 Finkelstein M, Liss GM, Krammer F, Kusiak RA. Mortality among workers receiving compensation awards for silicosis in Ontario 1940-85. Br J Ind Med 1987;44:588-94.

16 Zambon P, Simonato L, Mastrangelo G, Winkelmann R, Saia B, Crepet M. Mortality of workers compensated for silicosis during the period 1959-1963 in the Veneto region of Italy. Scand J Work Environ Health 1987;13:118-23.

17 Mastrangelo G, Zambon P, Simonato L, Rizzi P. A case-referent study investigating the relationship between exposure to silica 
dust and lung cancer. Int Arch Occup Environ Health 1988; 60:299-302.

18 Rimington J. Smoking, chronic bronchitis, and lung cancer. $\mathrm{Br}$ Med J 1971;2:373-5.

19 Davis AL. Bronchogenic carcinoma in chronic obstructive pulmonary disease. JAMA 1976;235:621-2.

20 Peto R, Speizer FE, Cochrane AL, et al. The relevance in adults of air-flow obstruction, but not of mucus hypersecretion, to mortality from chronic lung disease. Am Rev Respir Dis 1983;128:491-500.

21 Cohen BH, Ball WC, Brashears S, et al. Risk factors in chronic obstructive pulmonary disease (COPD). Am J Epidemiol 1977; 105:223-31.

22 Cohen $\mathrm{BH}$. Chronic obstructive pulmonary disease: a challenge in genetic epidemiology. Am J Epidemiol 1980;112:274-88.

23 Beaty TH, Cohen BH, Newill CA, Menkes HA, Diamond EL, Chen CJ. Impaired pulmonary function as a risk factor for mortality. Am J Epidemiol 1982;116:102-13.

24 Carta P, Aru G, Barbieri MT, et al. Cause di morte nei silicotici della Sardegna (1964-1977). Med Lav 1988;79:431-43.

25 International Labour Office. Guidelines for the use of ILO international classification of radiographs of pneumoconiosis. Geneva: ILO, 1980. (Occupational Safety and Health series No 22.)

26 Casula D. Aspetti diagnostici della silicosi (con particolare riguardo alla situazione in Sardegna). In: Proceedings of the second national conference on silicosis. Cagliari: 1965;1:25-60.

27 Casula D, Cherchi P, Sanna-Randaccio F, et al. In: Valdes P, ed. Piano di lotta contro la silicosi e le altre pneumoconiosi di particolare importanza per la Sardegna. Cagliari, 1982:1: 1-927.

28 Casula D, Carta P, Sanna-Randaccio F. A six year longitudinal study of Sardinian metalliferous miners. In: Proceedings of the VIth international conference on pneumoconiosis. Bochum. Geneva: International Labour Organisation, 1983;2:1107-12.

29 Rogan JM, Attfield MD, Jacobsen M, Rae S, Walker DD, Walton WH. Role of dust in the working environment in development of chronic bronchitis in British coal miners. $B r J$ Ind Med 1973;30:217-26.

30 Quanjer PH. Standardized lung function testing. Report of working party on standardization of lung function tests of the European Community for Coal and Steel. Bull Europ Physiopathol Respir 1983;19(suppl 5):1-94.

31 World Health Organisation: International Classification of Diseases. 8th rev. Geneva: WHO, 1967

32 Comba P, Belli S, Axelson O. Ricerche epidemiologiche in igiene e medicina del lavoro. Istituto Superiore di Sanita 1986;23:115-204.

33 Rothman KJ. Modern Epidemiology. Boston: Little Brown, 1986.

34 Lubin JH, Gail MH. Biased selection of controls for case-control analysis of cohort studies. Biometrics 1984;40:63-75.
35 Mantel N, Haenszel W. Statistical aspects of the analysis of data from retrospective studies of disease. J Nat Cancer Inst $1959 ; 22: 719-48$

36 Miettinen OS. Estimability and estimation in case-control studies. Am J Epidemiol 1976;103:226-35.

37 Hessel PA, Sluis-Cremer GK, Hnizdo E. Silica exposure, silicosis, and lung cancer: a necropsy study. $\mathrm{Br} J$ Ind Med 1990;47:4-9.

38 Jacobsen $\mathbf{M}$. Lung cancer and coal workers' pneumoconiosis. Br Med J 1979;2:208-12.

39 Rooke GB, Ward FG, Dempsey AN, Dowler JB, Whitaker CJ. Carcinoma of the lung in Lancashire coalminers. Thorax 1979;34:229-33.

40 Miller BG, Jacobsen M. Dust exposure, pneumoconiosis, and mortality of coalminers. Br J Ind Med 1985;42:723-33.

41 Dodgson J, Hadden GG, Jones CO, Walton WH. Characteristics of the airborne dust in British coal mines. In: Walton WH, ed. Inhaled Particles. III. Old Woking: Unwin Bros, 1971: 757-81.

42 Duggan MJ, Soilleux PJ, Strong JL, Howell DM. The exposure of United Kingdom miners to radon. Br J Ind Med 1970; 27:106-9.

43 Ames RG. Does coal workers' pneumoconiosis predict lung cancer? Some evidence from a case control study. $J$ Soc Occup Med 1983;33:141-4.

44 Davis L, Wegman D, Monson R. Mortality experience of Vermont granite workers. Am J Ind Med 1983;4:705-23.

45 Steenland $\mathrm{K}$, Beaumont J. A proportionate mortality study of granite cutters. Am J Ind Med 1986;9:189-201.

46 Costello J, Graham WGB. Vermont granite workers' mortality study. Am J Ind Med 1988;13:483-97.

47 Koskela RS, Klockars M, Jarvinen E, Kolari PJ, Rossi A. Cancer mortality of granite workers. Scand JWork Environ Health 1987;13:26-31.

48 Harber P, Oren A, Moshenifar Z, Lew M. Obstructive airway disease as risk factor for asbestos-associated malignancy. J Occup Med 1986;28:82-6.

49 Skillrud DM, Offord KP, Miller RD. Higher risk of lung cancer in chronic obstructive pulmonary disease. Ann Intern Med 1986; 105:503-7.

50 Tockman MS, Anthonisen NR, Wright EC, Donithan MG. IPPB trial group, Johns Hopkins lung project. Airway obstruction and the risk for lung cancer. Ann Intern Med 1987; 106:512-8.

51 Anthonisen NR. Prognosis in chronic obstructive pulmonary disease: results from multicenter clinical trials. Am Rev Respir Dis 1989;140:S95-S99.

$52 \mathrm{McDonald} \mathrm{JC}$. Silica, silicosis, and lung cancer. $\mathrm{Br} \mathrm{J}$ Ind Med 1989;46:289-91.

Accepted 20 August 1990 